Published in final edited form as:

Nature. 2021 May ; 593(7858): 249-254. doi:10.1038/s41586-021-03506-2.

\title{
High-performance brain-to-text communication via handwriting
}

\author{
Francis R. Willett ${ }^{1,2,3}$, Donald T. Avansino ${ }^{1}$, Leigh R. Hochberg ${ }^{4,5,6}$, Jaimie M. \\ Henderson $2,9,{ }^{*}$, Krishna V. Shenoy $1,3,7,8,9,{ }^{*}$ \\ ${ }^{1}$ Howard Hughes Medical Institute at Stanford University, Stanford, CA, USA \\ 2 Department of Neurosurgery, Stanford University, Stanford, CA, USA \\ ${ }^{3}$ Department of Electrical Engineering, Stanford University, Stanford, CA, USA \\ 4 VA RR\&D Center for Neurorestoration and Neurotechnology, Rehabilitation R\&D Service, \\ Providence VA Medical Center, Providence, RI, USA \\ ${ }^{5}$ School of Engineering and Carney Institute for Brain Science, Brown University, Providence, RI, \\ USA \\ ${ }^{6}$ Center for Neurotechnology and Neurorecovery, Department of Neurology, Massachusetts \\ General Hospital, Harvard Medical School, Boston, MA, USA \\ 7 Department of Bioengineering, Stanford University, Stanford, CA, USA \\ ${ }^{8}$ Department of Neurobiology, Stanford University, Stanford, CA, USA \\ ${ }^{9}$ Wu Tsai Neurosciences Institute and Bio-X Institute, Stanford University, Stanford, CA, USA
}

\section{Summary:}

Brain-computer interfaces (BCIs) can restore communication to people who have lost the ability to move or speak. To date, a major focus of $\mathrm{BCI}$ research has been on restoring gross motor skills, such as reaching and grasping ${ }^{1-5}$ or point-and-click typing with a $2 \mathrm{D}$ computer cursor ${ }^{6,7}$.

However, rapid sequences of highly dexterous behaviors, such as handwriting or touch typing, might enable faster communication rates. Here, we demonstrate an intracortical BCI that decodes attempted handwriting movements from neural activity in motor cortex and translates it to text in real-time, using a novel recurrent neural network decoding approach. With this BCI, our study

\footnotetext{
Corresponding author: Francis R. Willett (fwillett@stanford.edu).

*Co-senior author

Author Contributions

F.R.W. conceived the study, built the real-time decoder, analyzed the data, and wrote the manuscript. F.R.W. and D.T.A. collected the data. L.R.H. is the sponsor-investigator of the multi-site clinical trial. J.M.H. planned and performed T5's array placement surgery and was responsible for his ongoing clinical care. J.M.H. and K.V.S. supervised and guided the study. All authors reviewed and edited the manuscript.

Additional Information

Supplementary Information is available for this paper. Reprints and permissions information is available at www.nature.com/reprints.

Data Availability Statement

All neural data needed to reproduce the findings in this study are publicly available at the Dryad repository (https://doi.org/10.5061/ dryad.wh70rxwmv). The dataset contains neural activity recorded during attempted handwriting of 1,000 sentences (43.5k characters) over 10.7 hours.

Code Availability Statement

Code that implements an offline reproduction of the central findings in this study (high-performance neural decoding with an RNN) is publicly available on GitHub at https://github.com/fwillett/handwritingBCI.
} 
participant, whose hand was paralyzed from spinal cord injury, achieved typing speeds that exceed those of any other BCI yet reported: 90 characters per minute at $94.1 \%$ raw accuracy online, and $>99 \%$ accuracy offline with a general-purpose autocorrect. These speeds are comparable to ablebodied smartphone typing speeds in our participant's age group (115 characters per minute $)^{8}$. Finally, new theoretical considerations explain why temporally complex movements, such as handwriting, may be fundamentally easier to decode than point-to-point movements. Our results open a new approach for BCIs and demonstrate the feasibility of accurately decoding rapid, dexterous movements years after paralysis.

\section{Neural representation of handwriting}

Prior BCI studies have shown that the motor intention for gross motor skills, such as reaching, grasping or moving a computer cursor, remains neurally encoded in motor cortex after paralysis ${ }^{1-7}$. However, it is still unknown whether the neural representation for a rapid and highly-dexterous motor skill, such as handwriting, also remains intact. We tested this by recording neural activity from two microelectrode arrays in the hand "knob" area of precentral gyrus (a premotor area) $)^{9,10}$ while our BrainGate study participant, T5, attempted to handwrite individual letters and symbols (Fig. 1a). T5 has a high-level spinal cord injury (C4 AIS C) and was paralyzed from the neck down; his hand movements were entirely nonfunctional and limited to twitching and micromotion. We instructed T5 to "attempt" to write as if his hand was not paralyzed, while imagining that he was holding a pen on a piece of ruled paper.

To visualize the neural activity (multiunit threshold crossing rates), we used principal components analysis to display the top 3 neural dimensions containing the most variance

(Fig. 1b). The neural activity appeared to be strong and repeatable, although the timing of its peaks and valleys varied across trials, potentially due to fluctuations in writing speed. We used a time-alignment technique to remove temporal variability ${ }^{11}$, revealing remarkably consistent underlying patterns of neural activity that are unique to each character (Fig. 1c). To see if the neural activity encoded the pen movements needed to draw each character's shape, we attempted to reconstruct each character by linearly decoding pen tip velocity from the trial-averaged neural activity (Fig. 1d). Readily recognizable letter shapes confirm that pen tip velocity is robustly encoded. The neural dimensions that represented pen tip velocity accounted for $30 \%$ of the total neural variance.

Next, we used a nonlinear dimensionality reduction method (t-SNE) to produce a 2dimensional visualization of each single trial's neural activity recorded after the 'go' cue was given (Fig. 1e). The t-SNE visualization revealed tight clusters of neural activity for each character and a predominantly motoric encoding where characters that are written similarly are closer together. Using a k-nearest neighbor classifier applied offline to the neural activity, we could classify the characters with $94.1 \%$ accuracy $(95 \% \mathrm{CI}=[92.6$, 95.8]). Taken together, these results suggest that, even years after paralysis, the neural representation of handwriting in motor cortex is likely strong enough to be useful for a BCI. 


\section{Decoding handwritten sentences}

Next, we tested whether we could decode complete handwritten sentences in real-time, thus enabling a person with tetraplegia to communicate by attempting to handwrite their intended message. To do so, we trained a recurrent neural network (RNN) to convert the neural activity into probabilities describing the likelihood of each character being written at each moment in time (Fig. 2a, Extended Data Fig. 1). These probabilities could either be thresholded in a simple way to emit discrete characters, which we did for real-time decoding (Fig. 2a "Raw Online Output"), or processed more extensively by a large-vocabulary language model to simulate an autocorrect feature, which we applied offline (Fig. 2a "Offline Output from a Language Model"). We used the limited set of 31 characters shown in Fig. 1d, consisting of the 26 lower case letters of the alphabet, commas, apostrophes, question marks, periods (written by T5 as ' $\sim$ ') and spaces (written by T5 as '>'). The ' $\sim$ ' and '> ' symbols were chosen to make periods and spaces easier to detect. T5 attempted to write each character in print (not cursive), with each character printed on top of the previous one.

To collect training data for the RNN, we recorded neural activity while T5 attempted to handwrite complete sentences at his own pace, following instructions on a computer monitor. Prior to the first day of real-time evaluation, we collected a total of 242 sentences across 3 pilot days that were combined to train the RNN. On each subsequent day of realtime testing, additional training data were collected to recalibrate the RNN prior to evaluation, yielding a combined total of 572 training sentences by the last day (comprising 7.3 hours and $30.4 \mathrm{k}$ characters). To train the RNN, we adapted neural network methods in automatic speech recognition ${ }^{12-14}$ to overcome two key challenges: (1) the time that each letter was written in the training data was unknown (since T5's hand was paralyzed), making it challenging to apply supervised learning techniques, and (2) the dataset was limited in size compared to typical RNN datasets, making it difficult to prevent overfitting to the training data (see Supplemental Methods, Extended Data Figs. 2-3).

We evaluated the RNN's performance over a series of 5 days, each day containing 4 evaluation blocks of 7-10 sentences that the RNN was never trained on (thus ensuring that the RNN could not have overfit to those sentences). T5 copied each sentence from an onscreen prompt, attempting to handwrite it letter by letter, while the decoded characters appeared on the screen in real-time as they were detected by the RNN (Supplementary Video 1-2, Extended Data Table 1). Characters appeared after they were completed by T5 with a short delay (estimated to be $0.4-0.7$ seconds). The decoded sentences were quite legible (Fig. 2b, "Raw Output"). Importantly, typing rates were high, plateauing at 90 characters per minute with a $5.4 \%$ character error rate (Fig. 2c, average of red circles). Since there was no "backspace" function implemented, T5 was instructed to continue writing if any decoding errors occurred.

When a language model was used to autocorrect errors offline, error rates decreased considerably (Fig. 2c, open squares below filled circles; Table 1). The character error rate fell to $0.89 \%$ and the word error rate fell to $3.4 \%$ averaged across all days, which is comparable to state-of-the-art speech recognition systems (e.g. word error rates of 4-5\% 14,15 ), putting it well within the range of usability. Finally, to probe the limits of possible 
decoding performance, we trained a new RNN offline using all available sentences to process the entire sentence in a non-causal way (comparable to other BCI studies ${ }^{16,17}$ ). Accuracy was extremely high in this regime $(0.17 \%$ character error rate), indicating a high potential ceiling of performance, although this decoder would not be able to provide letterby-letter feedback to the user.

Next, to evaluate performance in a less restrained setting, we collected two days of data in which T5 used the BCI to freely type answers to open-ended questions (Supplementary Video 3, Extended Data Table 2). The results confirm that high performance can also be achieved when the user writes self-generated sentences as opposed to copying on-screen prompts (73.8 characters per minute with an $8.54 \%$ character error rate in real-time, $2.25 \%$ with a language model). The prior state-of-the-art for free typing in intracortical BCIs was 24.4 correct characters per minute ${ }^{7}$.

\section{Daily decoder retraining}

Following standard practice (e.g. ${ }^{1,2,18,4,5}$ ), we retrained our handwriting decoder each day before evaluating it, with the help of 'calibration' data collected at the beginning of each day. Retraining helps account for changes in neural recordings that accrue over time, which might be caused by neural plasticity or electrode array micromotion. Ideally, to reduce the burden on the user, little or no calibration data would be required. In a retrospective analysis of the copy typing data reported above in Fig. 2, we assessed whether high performance could still have been achieved using less than the original 50 calibration sentences per day (Fig. 3a). We found that 10 sentences ( 8.7 minutes) were enough to achieve a raw error rate of $8.5 \%$ ( $1.7 \%$ with a language model), although 30 sentences were needed to match the raw online error rate of $5.9 \%$.

However, our copy typing data were collected over a 28-day time span, possibly allowing larger changes in neural activity to accumulate. Using further offline analyses, we assessed whether more closely-spaced sessions reduce the need for calibration data (Fig. 3b). We found that when only 2-7 days passed between sessions, performance was reasonable with no decoder retraining ( $11.1 \%$ raw error rate, $1.5 \%$ with a language model), as might be expected from prior work showing short-term stability of neural recordings ${ }^{19-21}$. Finally, we tested whether decoders could be retrained in an unsupervised manner by using a language model to error-correct and retrain the decoder, thus bypassing the need to interrupt the user for calibration (i.e., by recalibrating automatically during normal use). Encouragingly, unsupervised retraining achieved a $7.3 \%$ raw error rate $(0.84 \%$ with a language model) when sessions were separated by 7 days or less.

Ultimately, whether decoders can be successfully retrained with minimal recalibration data depends on how quickly the neural activity changes over time. We assessed the stability of the neural patterns associated with each character and found high short-term stability (mean correlation $=0.85$ when 7 days apart or less), and neural changes that seemed to accumulate at a steady and predictable rate (Extended Data Fig. 4). The above results are promising for clinical viability, as they suggest that unsupervised decoder retraining, combined with more limited supervised retraining after longer periods of inactivity, may be sufficient to achieve 
high performance. Nevertheless, future work must confirm this online, as offline simulations are not always predictive of online performance.

\section{Temporal variety improves decoding}

To our knowledge, 90 characters per minute is the highest typing rate yet reported for any type of BCI (see Discussion). For intracortical BCIs, the highest performing method has been point-and-click typing with a $2 \mathrm{D}$ computer cursor, peaking at 40 characters per minute

${ }^{7}$ (see Supplementary Video 4 for a direct comparison). The speed of point-and-click BCIs is limited primarily by decoding accuracy. During parameter optimization, the cursor gain is increased so as to increase typing rate, until the cursor moves so quickly that it becomes uncontrollable due to decoding errors ${ }^{22}$. How is it then that handwriting movements could be decoded more than twice as fast, with similar levels of accuracy?

We theorize that handwritten letters may be easier to distinguish from each other than pointto-point movements, since letters have more variety in their spatiotemporal patterns of neural activity than do straight-line movements. To test this theory, we analyzed the patterns of neural activity associated with 16 straight-line movements and 16 letters that required no lifting of the pen off the page, both performed by T5 with attempted handwriting (Fig. 4ab).

First, we analyzed the pairwise Euclidean distances between each neural activity pattern. We found that the nearest-neighbor distances for each movement were $72 \%$ larger for characters as compared to straight lines $(95 \% \mathrm{CI}=[60 \%, 86 \%])$, making it less likely for a decoder to confuse two nearby characters (Fig. 4c). To confirm this, we simulated the classification accuracy for each set of movements as a function of neural noise (Fig. 4d), demonstrating that characters are easier to classify than straight lines.

To gain insight into what might be responsible for the relative increase in nearest neighbor distances for characters, we examined the spatial and temporal dimensionality of the neural patterns. Spatial and temporal dimensionality were estimated using the "participation ratio", which quantifies approximately how many spatial or temporal dimensions are required to explain $80 \%$ of the variance in the neural activity patterns ${ }^{23}$. We found that spatial dimensionality was only modestly larger for characters $(1.24$ times larger; $95 \% \mathrm{CI}=[1.19$, $1.30])$, but that the temporal dimensionality was much greater $(2.65$ times larger; $95 \% \mathrm{CI}=$ $[2.58,2.72])$, suggesting that the increased variety of temporal patterns in letter writing drives the increased separability of each movement (Fig. 4e).

To illustrate how increased temporal dimensionality can make movements more distinguishable, we constructed a toy model with four movements and two neurons whose activity is constrained to lie along a single dimension (Fig. $4 \mathrm{f}-\mathrm{g}$ ). Simply by allowing the trajectories to change in time (Fig. 4g), the nearest neighbor distance between the neural trajectories can be increased, resulting in an increase in classification accuracy when noise levels are large enough (Fig. 4h). Although neural noise in the toy model was assumed to be independent white noise, we found that these results also hold for noise that is correlated across time and neurons (Extended Data Fig. 5 and Supplemental Note 1). 
These results suggest that time-varying patterns of movement, such as handwritten letters, are fundamentally easier to decode than point-to-point movements. We think this is one, but not necessarily the only, important factor that enabled a handwriting BCI to go faster than continuous-motion point-and-click BCIs. Other discrete (classification-based) BCIs have also typically used directional movements with little temporal variety, which may have limited their accuracy and/or the size of the movement $\operatorname{set}^{24,25}$.

More generally, using the principle of maximizing the nearest neighbor distance between movements, it should be possible to optimize a set of movements for ease of classification ${ }^{26}$. We explored doing so and designed an alphabet that is theoretically easier to classify than the Latin alphabet (Extended Data Fig. 6). The optimized alphabet avoids large clusters of redundant letters that are written similarly (most Latin letters begin with either a downstroke or a counter-clockwise curl).

\section{Discussion}

Locked-in syndrome (paralysis of nearly all voluntary muscles) severely impairs or prevents communication, and is most frequently caused by brainstem stroke or late-stage ALS (estimated prevalence of locked-in syndrome: 1 in $100,000{ }^{27}$ ). Commonly used BCIs for restoring communication are either flashing EEG spellers ${ }^{28-30,18,31,32}$ or intracortical pointand-click BCIs ${ }^{33,6,7}$. EEG spellers based on oddball potentials or motor imagery typically achieve 1-5 characters per minute ${ }^{28-32}$. EEG spellers that use visually evoked potentials have achieved speeds of 60 characters per minute ${ }^{18}$, but have important usability limitations, as they tie up the eyes, are not typically self-paced, and require panels of flashing lights on a screen. Intracortical BCIs based on 2D cursor movements give the user more freedom to look around and set their own pace of communication, but have yet to exceed 40 correct characters per minute in people ${ }^{7}$. Recently, speech-decoding BCIs have shown exciting promise for restoring rapid communication (e.g. ${ }^{34,16,17}$ ), but their accuracies and vocabulary sizes require further improvement to support general-purpose use.

Here, we introduced a novel approach for communication BCIs - decoding a rapid, dexterous motor behavior in a person with tetraplegia - that sets a new benchmark for communication rate at 90 characters per minute. The demonstrated real-time system is general (the user can express any sentence), easy to use (entirely self-paced and the eyes are free to move), and accurate enough to be useful in the real-world (94.1\% raw accuracy, and $>99 \%$ accuracy offline with a large-vocabulary language model). To achieve high performance, we developed new decoding methods to work effectively with unlabeled neural sequences in data-limited regimes. These methods could be applied more generally to any sequential behavior that cannot be observed directly (e.g., decoding speech from someone who can no longer speak).

Finally, it is important to recognize that the current system is a proof-of-concept that a highperformance handwriting $\mathrm{BCI}$ is possible (in a single participant); it is not yet a complete, clinically viable system. More work is needed to demonstrate high performance in additional people, expand the character set (e.g., capital letters), enable text editing and deletion, and maintain robustness to changes in neural activity without interrupting the user for decoder 
retraining. More broadly, intracortical microelectrode array technology is still maturing, and requires further demonstrations of longevity, safety, and efficacy before widespread clinical adoption $^{35,36}$. Variability in performance across participants is also a potential concern (in a prior study, T5 achieved the highest performance of 3 participants $^{7}$ ).

Nevertheless, we believe the future of intracortical BCIs is bright. Current microelectrode array technology has been shown to retain functionality for 1000+ days post implant ${ }^{37,38}$ (including here; see Extended Data Fig. 7), and has enabled the highest BCI performance to date compared to other recording technologies (EEG, ECoG) for restoring communication ${ }^{7}$, arm control ${ }^{2,5}$, and general-purpose computer use ${ }^{39}$. New developments are underway for implant designs that increase the electrode count by at least an order of magnitude, which will further improve performance and longevity $35,36,40,41$. Finally, we envision that a combination of algorithmic innovations ${ }^{42-44}$ and improvements to device stability will continue to reduce the need for daily decoder retraining. Here, offline analyses showed the potential promise of more limited, or even unsupervised, decoder retraining (Fig. 3).

\section{Extended Data}

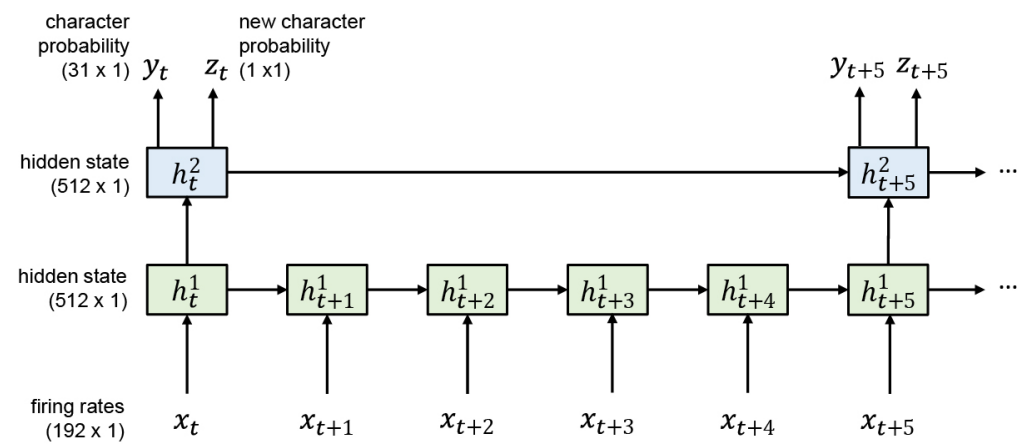

Extended Data Fig. 1: Diagram of the RNN architecture.

We used a two-layer gated recurrent unit (GRU) recurrent neural network architecture to convert sequences of neural firing rate vectors $x_{t}$ (which were temporally smoothed and binned at $20 \mathrm{~ms}$ ) into sequences of character probability vectors $y_{t}$ and 'new character' probability scalars $z_{t}$. The $y_{t}$ vectors describe the probability of each character being written at that moment in time, and the $z_{t}$ scalars go high whenever the RNN detects that T5 is beginning to write any new character. Note that the top RNN layer runs at a slower frequency than the bottom layer, which we found improved the speed of training by making it easier to hold information in memory for long time periods. Thus, the RNN outputs are updated only once every $100 \mathrm{~ms}$. 
a

Copy Typing Session Flow (Sessions 3-9)

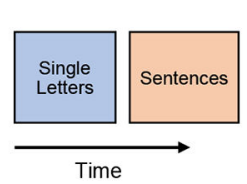

Time

Training Data Collection ( 1 - 1.5 hours)

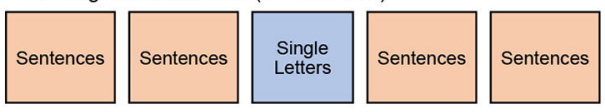

Free Typing Session Flow (Sessions 10-11)
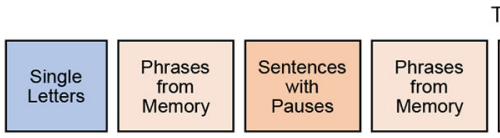

Training Data Collection ( 1.5 hours)
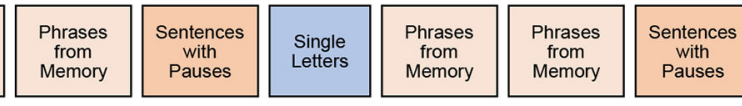

Main Training Steps

b

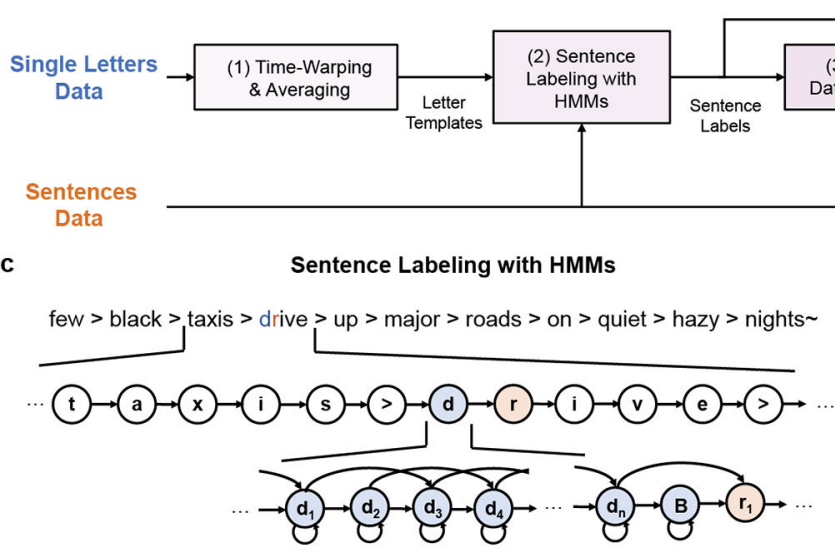

e

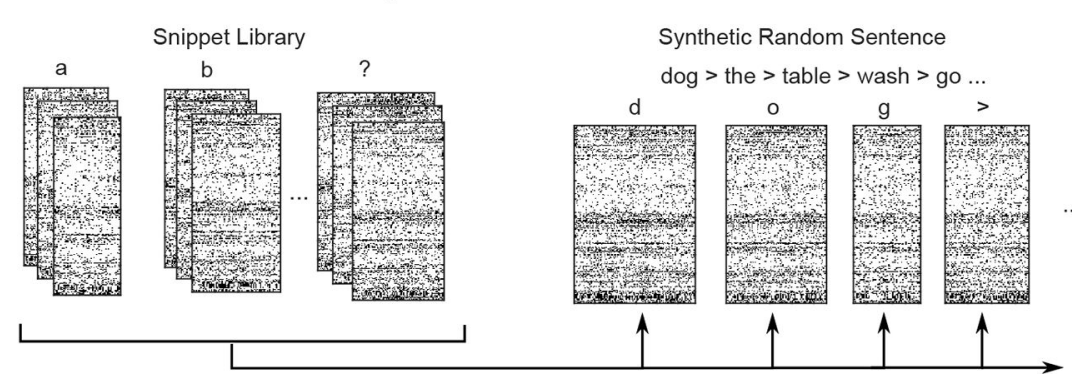

d
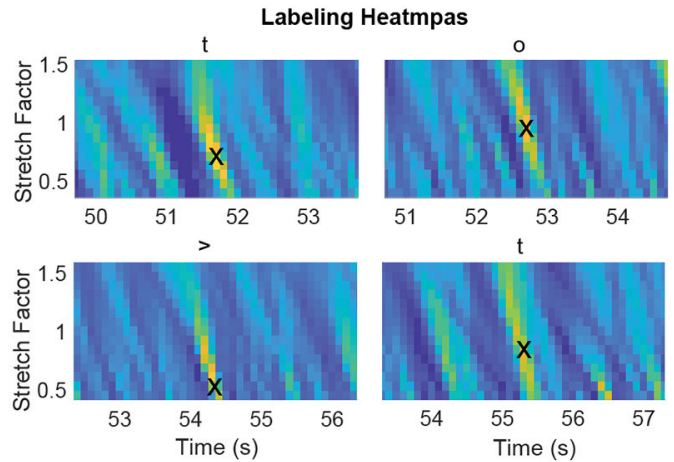

Correlation

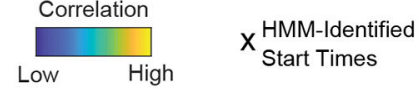

\section{Extended Data Fig. 2: Overview of RNN training methods.}

a, Diagram of the session flow for copy typing and free typing sessions (each rectangle corresponds to one block of data). First, single letter and sentences training data is collected (blue and red blocks). Next, the RNN is trained using the newly collected data plus all previous days' data (purple block). Finally, the RNN is held fixed and evaluated (green blocks). $\mathbf{b}$, Diagram of the data processing and RNN training process (purple block in a). First, the single letter data is time-warped and averaged to create spatiotemporal templates of neural activity for each character. These templates are used to initialize the hidden Markov 
models (HMMs) for sentence labeling. After labeling, the observed data is cut apart and rearranged into new sequences of characters to make synthetic sentences. Finally, the synthetic sentences are combined with the real sentences to train the RNN. c, Diagram of a forced-alignment HMM used to label the sentence "few black taxis drive up major roads on quiet hazy nights". The HMM states correspond to the sequence of characters in the sentence. d, The label quality can be verified with cross-correlation heatmaps made by correlating the single character neural templates with the real data. The HMM-identified character start times form clear hotspots on the heatmaps. Note that these heatmaps are depicted only to qualitatively show label quality and aren't used for training (only the character start times are needed to generate the targets for RNN training). e, To generate new synthetic sentences, the neural data corresponding to each labeled character in the real data is cut out of the data stream and put into a snippet library. These snippets are then pulled from the library at random, stretched/compressed in time by up to $30 \%$ (to add more artificial timing variability), and combined into new sentences.

a

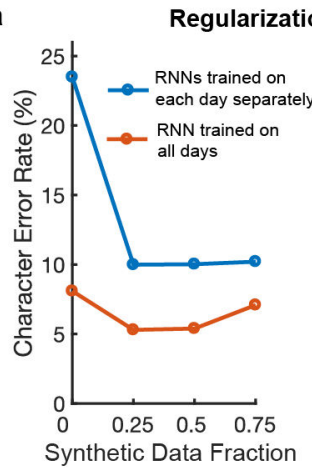

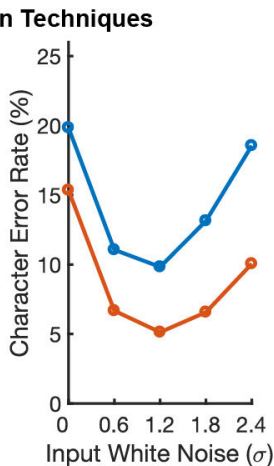

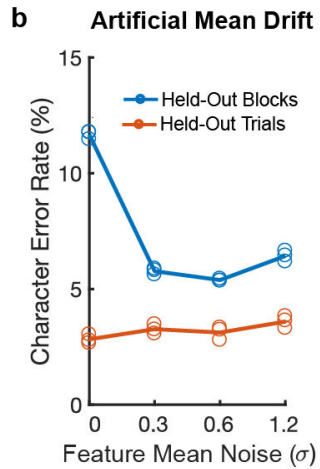

c

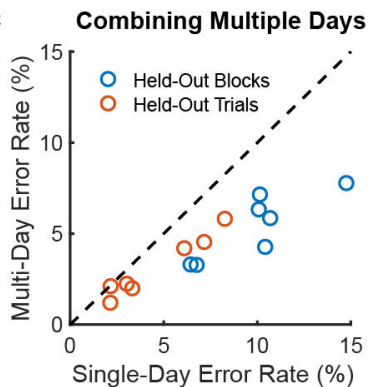

d Day-Specific Input Layers

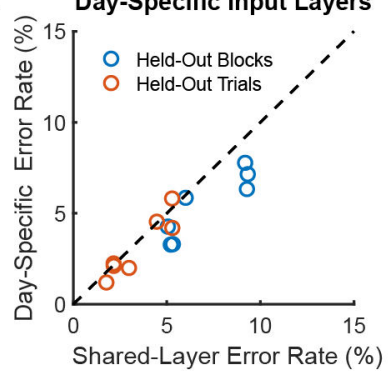

\begin{tabular}{|c|c|c|c|}
\hline Parameter & Panel & Comparison & $\begin{array}{l}\text { Error Rate Improvement } \\
{[95 \% \mathrm{Cl}]}\end{array}$ \\
\hline \multirow[t]{2}{*}{ Synthetic data fraction } & \multirow[t]{2}{*}{ a (left) } & Single-day RNN (0 vs. 0.25) & $12.9[11.9,14.0]$ \\
\hline & & Multi-day RNN (0 vs. 0.25 ) & $2.7[2.2,3.2]$ \\
\hline \multirow[t]{2}{*}{ Input white noise } & \multirow[t]{2}{*}{ a (right) } & Single-day RNN (0 vs. 1.2) & $9.8[8.7,11.0]$ \\
\hline & & Multi-day RNN (0 vs. 1.2) & $10.1[9.2,11.1]$ \\
\hline \multirow[t]{2}{*}{ Feature mean noise } & \multirow[t]{2}{*}{$b$} & Held-out blocks (0 vs. 0.3 ) & $5.7[4.9,6.5]$ \\
\hline & & Held-out trials (0 vs. 0.3 ) & $-0.7[-1.3,-0.1]$ \\
\hline \multirow[t]{2}{*}{ Single-day vs. multi-day } & \multirow[t]{2}{*}{$c$} & Held-out blocks & $4.7[4.1,5.3]$ \\
\hline & & Held-out trials & $1.5[0.6,2.4]$ \\
\hline \multirow{2}{*}{$\begin{array}{l}\text { Shared-layer vs. day- } \\
\text { specific layers }\end{array}$} & \multirow[t]{2}{*}{$d$} & Held-out blocks & $1.6[1.1,2.7]$ \\
\hline & & Held-out trials & $0.1[-0.7,0.7]$ \\
\hline
\end{tabular}

Nature. Author manuscript; available in PMC 2021 November 12. 
Extended Data Fig. 3: The effect of key RNN parameters on performance.

a, Training with synthetic data (left) and artificial white noise added to the inputs (right) were both essential for high performance. Data are shown from a grid search over both parameters, and lines show performance at the best value for the other parameter. Results indicate that both parameters are needed for high performance, even when the other is at the best value. Using synthetic data is more important when the dataset size is highly limited, as is the case when training on only a single day of data (blue line). Note that the inputs given to the RNN were z-scored, so the input white noise is in units of standard deviations of the input features. b, Artificial noise added to the feature means (random offsets and slow changes in the baseline firing rate) greatly improves the RNN's ability to generalize to new blocks of data that occur later in the session, but does not help the RNN to generalize to new trials within blocks of data that it was already trained on. This is because feature means change slowly over time. For each parameter setting, three separate RNNs were trained (circles); results show low variability across RNN training runs. c, Training an RNN with all of the datasets combined improves performance relative to training on each day separately. Each circle shows the performance on one of seven days. d, Using separate input layers for each day is better than using a single layer across all days. e, Improvements in character error rates are summarized for each parameter. 95\% confidence intervals were computed with bootstrap resampling of single trials $(\mathrm{N}=10,000)$. As shown in the table, all parameters show a statistically significant improvement for at least one condition (confidence intervals do not intersect zero). 
a

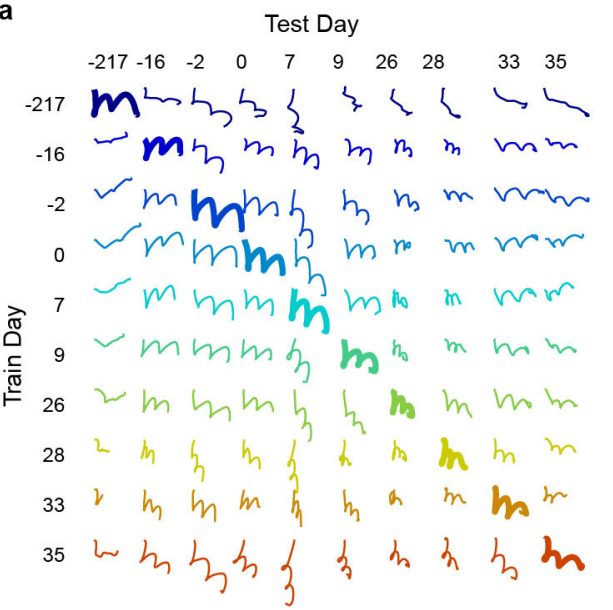

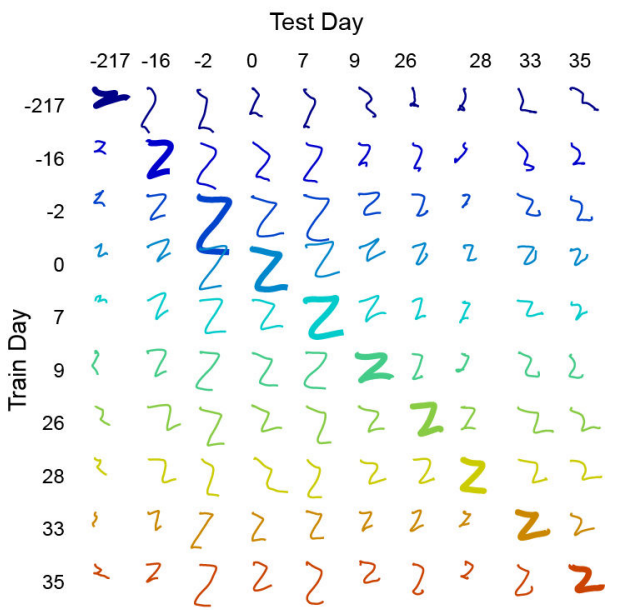

b

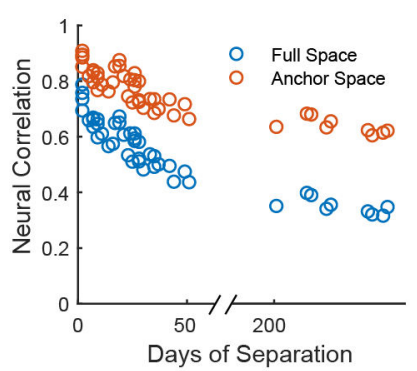

d

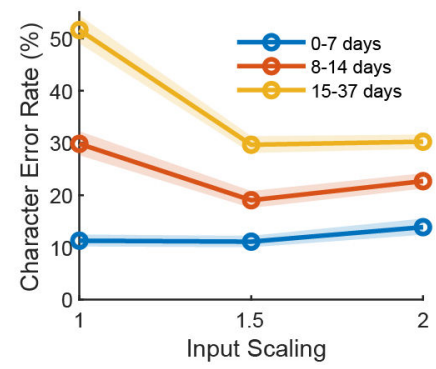

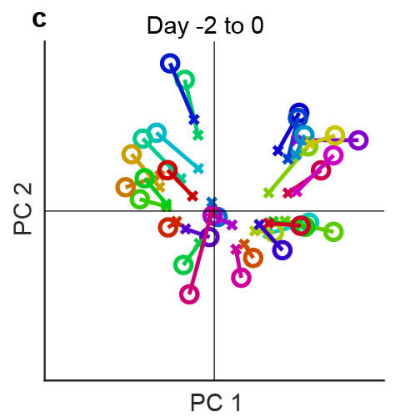

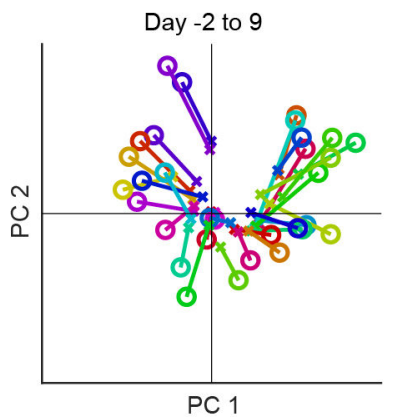

Extended Data Fig. 4: Changes in neural recordings across days.

a, To visualize how much the neural recordings changed across time, decoded pen tip trajectories were plotted for two example letters (" $\mathrm{m}$ " and " $\mathrm{z}$ ") for all ten days of data (columns), using decoders trained on all other days (rows). Each session is labeled according to the number of days passed relative to Dec. 9, 2019 (day \#4). Results show that although neural activity patterns clearly change over time, their essential structure is largely conserved (since decoders trained on past days transfer readily to future days). b, The correlation (Pearson's $r$ ) between each session's neural activity patterns was computed for each pair of sessions and plotted as a function of the number of days separating each pair.

Blue circles show the correlation computed in the full neural space (all 192 electrodes) while red circles show the correlation in the "anchor" space (top 10 principal components of the earlier session). High values indicate a high similarity in how characters are neurally encoded across days. The fact that correlations are higher in the anchor space suggests that the structure of the neural patterns stays largely the same as it slowly rotates into a new 
space, causing shrinkage in the original space but little change in structure. c, A visualization of how each character's neural representation changes over time, as viewed through the top two PCs of the original "anchor" space. Each "o" represents the neural activity pattern for a single character, and each " $x$ " shows that same character on a later day (lines connect matching characters). The left panel shows a pair of sessions with only two days between them ("Day -2 to 0 "), while the right panel shows a pair of sessions with 11 days between them ("Day -2 to 9"). The relative positioning of the neural patterns remains similar across days, but most conditions shrink noticeably towards the origin. This is consistent with the neural representations slowly rotating out of the original space into a new space, and suggests that scaling-up the input features may help a decoder to transfer more accurately to a future session (by counteracting this shrinkage effect). d, Similar to Fig. 3b, copy typing data from eight sessions was used to assess offline whether scaling-up the decoder inputs improves performance when evaluating the decoder on a future session (when no decoder retraining is employed). All session pairs (X, Y) were considered. Decoders were first initialized using all data from session $\mathrm{X}$ and earlier, then evaluated on session $\mathrm{Y}$ under different input scaling factors (e.g., an input scale of 1.5 means that input features were scaled up by 50\%). Lines indicate the average raw character error rate and shaded regions show 95\% CIs. Results show that when long periods of time pass between sessions, input-scaling improves performance. We therefore used an input scaling factor of 1.5 when assessing decoder performance in the "no retraining" conditions of Fig. 3.

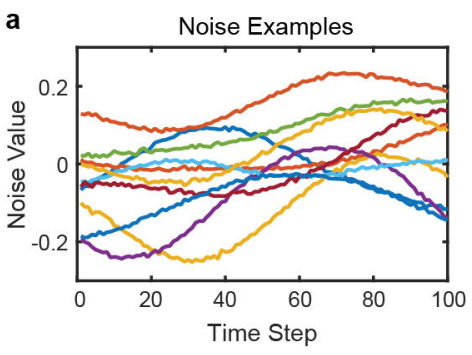

\section{Temporally Correlated Noise}
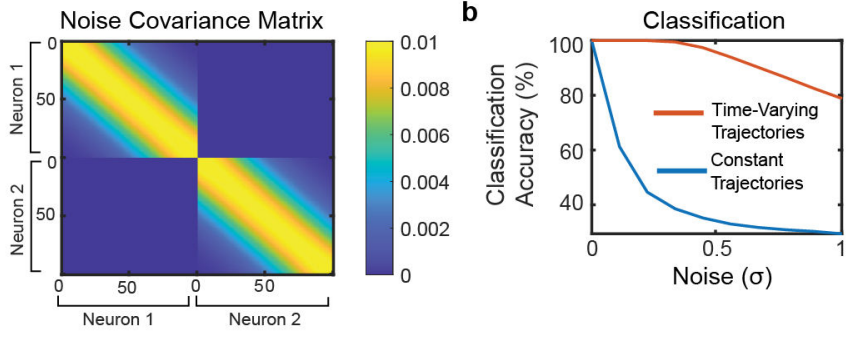

Signal-Correlated Noise

c

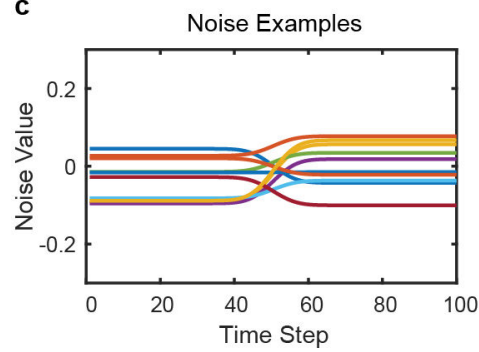

Noise Covariance Matrix

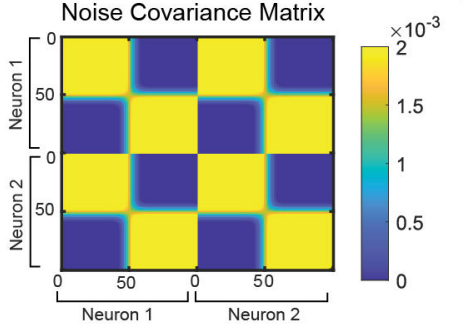

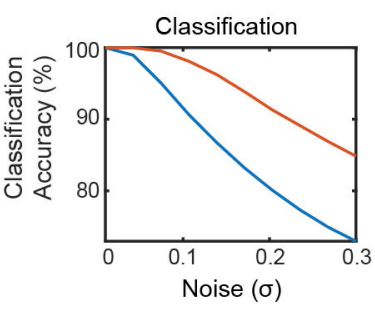

Extended Data Fig. 5: Effect of correlated noise on the toy model of temporal dimensionality (see Supplemental Note 1 for a detailed interpretation of this figure).

a, Example noise vectors and covariance matrix for temporally correlated noise. On the left, example noise vectors are plotted (each line depicts a single example). Noise vectors are shown for all 100 time steps of neuron 1. On the right, the covariance matrix used to generate temporally correlated noise is plotted (dimensions $=200 \times 200$ ). The first 100 time steps describe neuron 1's noise and the last 100 time steps describe neuron 2's noise. The 
diagonal band creates noise that is temporally correlated within each simulated neuron (but the two neurons are uncorrelated with each other). $\mathbf{b}$, Classification accuracy when using a maximum likelihood classifier to classify between all four possible trajectories in the presence of temporally correlated noise. Even in the presence of temporally correlated noise, the time-varying trajectories are still much easier to classify. c, Example noise vectors and noise covariance matrix for noise that is correlated with the signal (i.e., noise that is concentrated only in spatiotemporal dimensions that span the class means). Unlike the temporally correlated noise, this covariance matrix generates spatiotemporal noise that has correlations between time steps and neurons. $\mathbf{d}$, Classification accuracy in the presence of signal-correlated noise. Again, time-varying trajectories are easier to classify than constant trajectories.

a

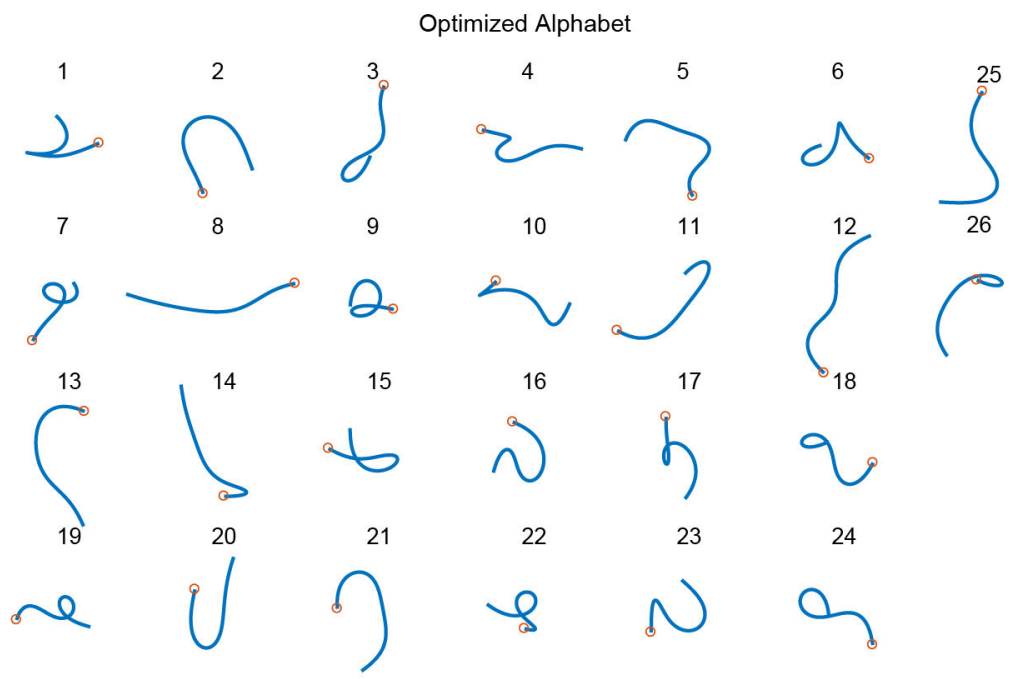

b

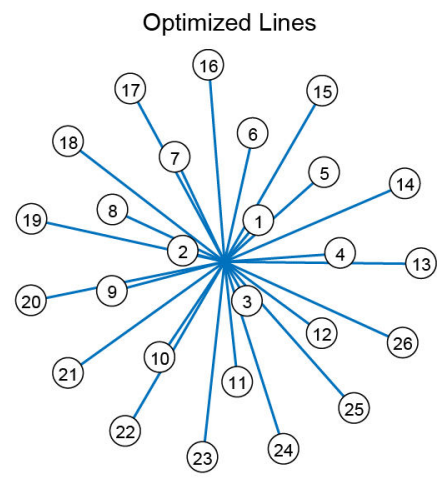

C
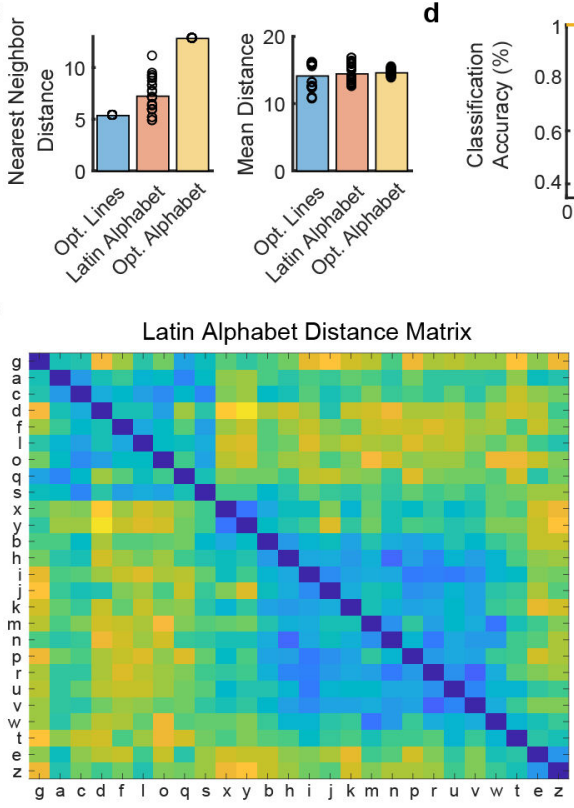

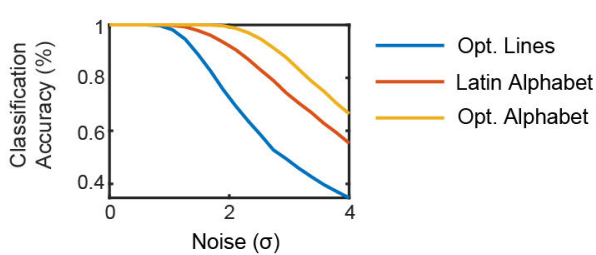

d

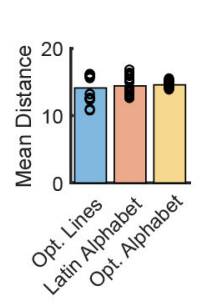

Nose

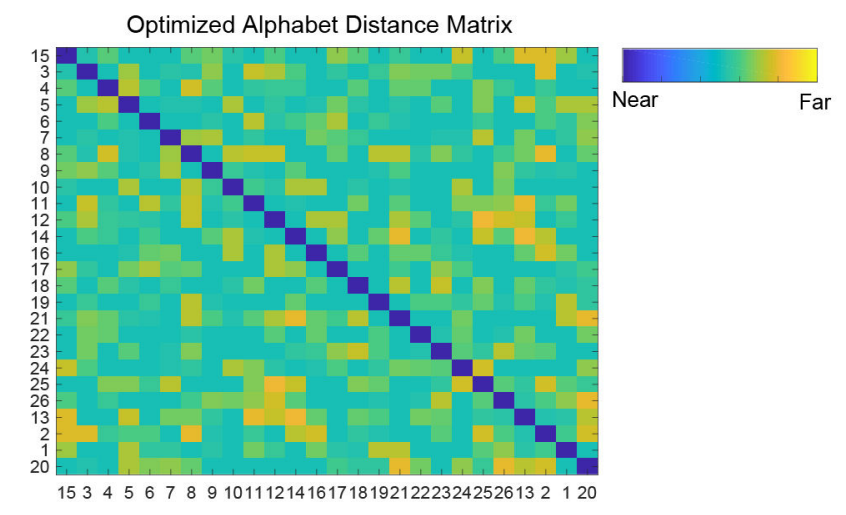

Extended Data Fig. 6: An artificial alphabet optimized to maximize neural decodability. 
(A) Using the principle of maximizing the nearest neighbor distance, we optimized for a set of pen trajectories that are theoretically easier to classify than the Latin alphabet (using standard assumptions of linear neural tuning to pen tip velocity). (B) For comparison, we also optimized a set of 26 straight lines that maximize the nearest neighbor distance. (C) Pairwise Euclidean distances between pen tip trajectories were computed for each set, revealing a larger nearest neighbor distance (but not mean distance) for the optimized alphabet as compared to the Latin alphabet. Each circle represents a single movement and bar heights show the mean. (D) Simulated classification accuracy as a function of the amount of artificial noise added. Results confirm that the optimized alphabet is indeed easier to classify than the Latin alphabet, and that the Latin alphabet is much easier to classify than straight lines, even when the lines have been optimized. (E) Distance matrices for the Latin alphabet and optimized alphabets show the pairwise Euclidean distances between the pen trajectories. The distance matrices were sorted into 7 clusters using single-linkage hierarchical clustering. The distance matrix for the optimized alphabet has no apparent structure; in contrast, the Latin alphabet has two large clusters of similar letters (letters that begin with a counter-clockwise curl, and letters that begin with a down stroke).
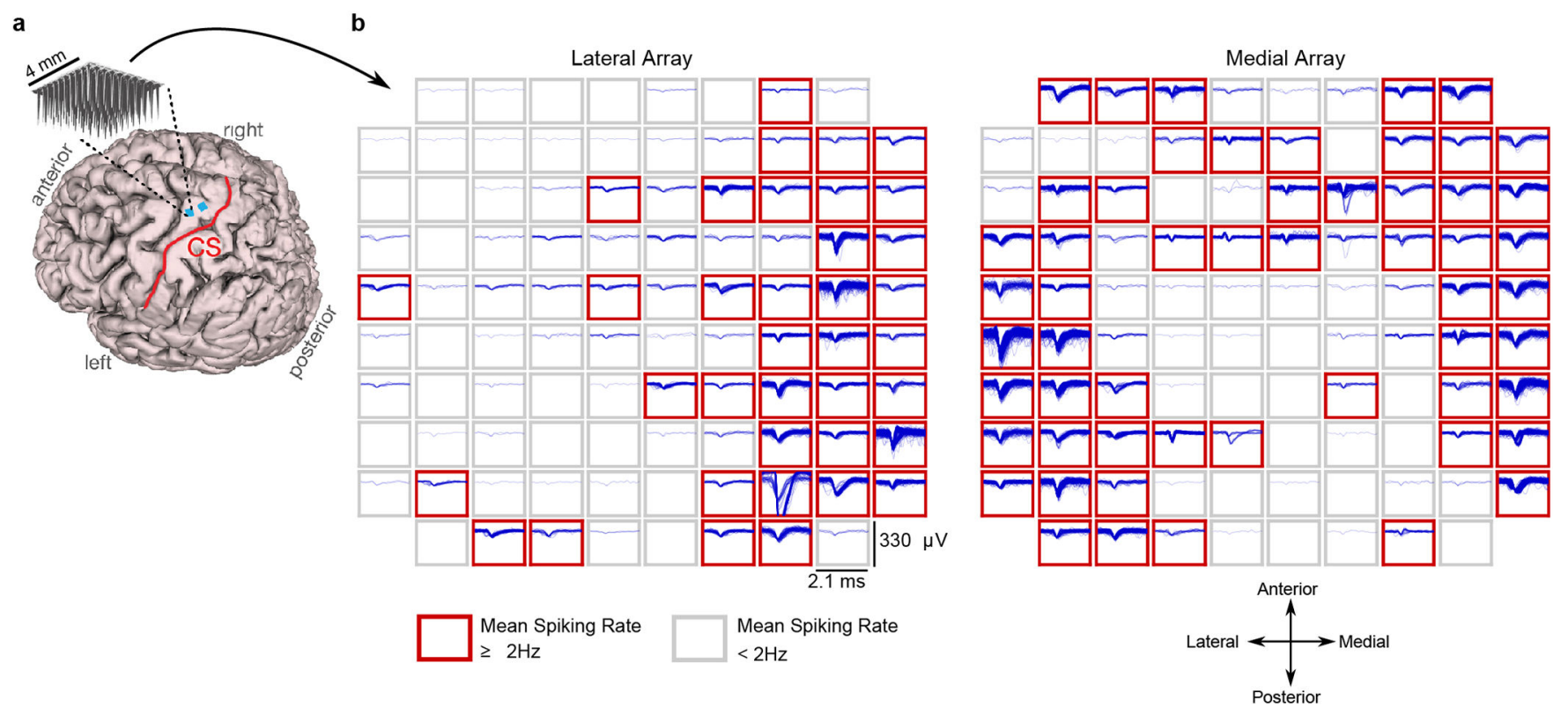

Extended Data Fig. 7: Example spiking activity recorded from each microelectrode array. (A) Participant T5's MRI-derived brain anatomy. Microelectrode array locations (blue squares) were determined by co-registration of postoperative CT images with preoperative MRI images. (B) Example spike waveforms detected during a ten second time window are plotted for each electrode (data were recorded on post-implant day 1218). Each rectangular panel corresponds to a single electrode and each blue trace is a single spike waveform (2.1 millisecond duration). Spiking events were detected using a -4.5 RMS threshold, thereby excluding almost all background activity. Electrodes with a mean threshold crossing rate $\geq 2$ $\mathrm{Hz}$ were considered to have 'spiking activity' and are outlined in red (note that this is a conservative estimate that is meant to include only spiking activity that could be from single neurons, as opposed to multiunit 'hash'). Results show that many electrodes still record large 
spiking waveforms that are well above the noise floor (the y-axis of each panel spans 330 $\mu \mathrm{V}$, while the background activity has an average RMS value of only $6.4 \mu \mathrm{V})$. On this day, 92 electrodes out of 192 had a threshold crossing rate $\geq 2 \mathrm{~Hz}$.

\section{Extended Data Table 1:}

Example decoded sentences for one block of copy typing. In the rightmost columns, errors are highlighted in red (extra spaces were denoted with a red square, and omitted letters were indicated with a strikethrough). Note that our language model substitutes "epidermal" for "epidural", since "epidural" is out of vocabulary. The mean characters per minute for this block was 86.47 and the character error rates were $4.18 \%$ (real-time output) and $1.22 \%$ (language model). Sentence prompts were taken from the BNC corpus according to a random selection process (see Supplementary Methods for details).

\begin{tabular}{|c|c|c|c|}
\hline Prompt & Real-Time Output & $\begin{array}{l}\text { Real-Time Output } \\
\text { (Annotated) }\end{array}$ & $\begin{array}{l}\text { Offline Language } \\
\text { Model (Annotated) }\end{array}$ \\
\hline $\begin{array}{l}\text { infected adults } \\
\text { develop a cough and } \\
\text { their skin and ears } \\
\text { turn blue. }\end{array}$ & $\begin{array}{l}\text { infected adults dercep a } \\
\text { cough and thhir skin } \\
\text { and ears tunn blue }\end{array}$ & $\begin{array}{l}\text { infected adults dercep a } \\
\text { cough and thhir skin } \\
\text { and ears tunn blue. }\end{array}$ & $\begin{array}{l}\text { infected adults develop } \\
\text { a cough and their skin } \\
\text { and ears turn blue. }\end{array}$ \\
\hline $\begin{array}{l}\text { i interrupted, unable to } \\
\text { keep silent. }\end{array}$ & $\begin{array}{l}\text { i interrupted, unabee to } \\
\text { keep silent. }\end{array}$ & $\begin{array}{l}\text { i interrupted, unabee to } \\
\text { keep silent. }\end{array}$ & $\begin{array}{l}\text { i interrupted, unable to } \\
\text { keep silent. }\end{array}$ \\
\hline $\begin{array}{l}\text { i dumped the tools in } \\
\text { the hut. }\end{array}$ & $\begin{array}{l}\text { i dumped the tools in } \\
\text { the hut. }\end{array}$ & $\begin{array}{l}\text { i dumped the tools in } \\
\text { the hut. }\end{array}$ & $\begin{array}{l}\text { i dumped the tools in } \\
\text { the hut. }\end{array}$ \\
\hline $\begin{array}{l}\text { within thirty seconds } \\
\text { the army had landed. }\end{array}$ & $\begin{array}{l}\text { within thirty seconds the } \\
\text { armmy had landed }\end{array}$ & $\begin{array}{l}\text { within thirty seconds the } \\
\text { armmy had landed. }\end{array}$ & $\begin{array}{l}\text { within thirty seconds the } \\
\text { army had landed. }\end{array}$ \\
\hline $\begin{array}{l}\text { that's when i threw up } \\
\text { on the carpet. }\end{array}$ & $\begin{array}{l}\text { that's when i threw up } \\
\text { on the carpet. }\end{array}$ & $\begin{array}{l}\text { that's when i threw up } \\
\text { on the carpet. }\end{array}$ & $\begin{array}{l}\text { that's when i threw up } \\
\text { on the carpet. }\end{array}$ \\
\hline $\begin{array}{l}\text { he didn't want to rub } \\
\text { salt into her wounds. }\end{array}$ & $\begin{array}{l}\text { he didn't want to rub salt } \\
\text { into her wounds. }\end{array}$ & $\begin{array}{l}\text { he didn't want to rub salt } \\
\text { into her wounds. }\end{array}$ & $\begin{array}{l}\text { he didn't want to rub salt } \\
\text { into her wounds. }\end{array}$ \\
\hline $\begin{array}{l}\text { shouting and } \\
\text { swearing, i yelled for } \\
\text { an epidural. }\end{array}$ & $\begin{array}{l}\text { shouting and sweerin, I } \\
\text { yeled for an epidural. }\end{array}$ & $\begin{array}{l}\text { shouting and sweering, i } \\
\text { yelled for } 1 \text { an epidural. }\end{array}$ & $\begin{array}{l}\text { shouting and swearing, i } \\
\text { yelled for a an } \\
\text { epidermal. }\end{array}$ \\
\hline $\begin{array}{l}\text { you wish to purchase } \\
\text { something? }\end{array}$ & $\begin{array}{l}\text { you wish to purchase } \\
\text { something? }\end{array}$ & $\begin{array}{l}\text { you wish to purchase } \\
\text { somathing? }\end{array}$ & $\begin{array}{l}\text { you wish to purchase } \\
\text { something? }\end{array}$ \\
\hline $\begin{array}{l}\text { lowell felt like a } \\
\text { soldier on a } \\
\text { battlefield, stripped of } \\
\text { ammunition. }\end{array}$ & $\begin{array}{l}\text { lowel felt like a soldier } \\
\text { on a battlefield, stripped } \\
\text { ef ammunition. }\end{array}$ & $\begin{array}{l}\text { lowell felt like a soldier } \\
\text { on a battlefield, stripped } \\
\text { ef ammunition. }\end{array}$ & $\begin{array}{l}\text { lowell felt like a soldier } \\
\text { on a battlefield, stripped } \\
\text { of ammunition. }\end{array}$ \\
\hline $\begin{array}{l}\text { there are only one or } \\
\text { two minor casualties. }\end{array}$ & $\begin{array}{l}\text { thee ane only on or two } \\
\text { minor cafualties. }\end{array}$ & $\begin{array}{l}\text { there ane only one or } \\
\text { two minor cafualties. }\end{array}$ & $\begin{array}{l}\text { there are only one or } \\
\text { two minor casualties. }\end{array}$ \\
\hline
\end{tabular}




\section{Extended Data Table 2:}

Example decoded sentences for one block of free typing. In the rightmost columns, errors are highlighted in red (omitted letters were indicated with a strikethrough). Note that our language model substitutes "salt fish" for "sailfish", since "sailfish" is out of vocabulary. The mean characters per minute for this block was 73.8 and the estimated character error rates were $6.82 \%$ (real-time output) and $1.14 \%$ (language model).

\begin{tabular}{|c|c|c|c|}
\hline Prompt & Real-Time Output & $\begin{array}{l}\text { Real-Time Output } \\
\text { (Annotated) }\end{array}$ & $\begin{array}{l}\text { Offline Language } \\
\text { Model (Annotated) }\end{array}$ \\
\hline $\begin{array}{l}\text { what made you first } \\
\text { get interested in } \\
\text { machining? }\end{array}$ & $\begin{array}{l}\text { iit was the famil } \\
\text { business }\end{array}$ & $\begin{array}{l}\text { iit was the family } \\
\text { business }\end{array}$ & $\begin{array}{l}\text { it was the family } \\
\text { business }\end{array}$ \\
\hline $\begin{array}{l}\text { what is the hardest } \\
\text { part of machining? }\end{array}$ & forming the tooling & forming the tooling & forming the tooling \\
\hline $\begin{array}{l}\text { how much spice do } \\
\text { you like in your food? }\end{array}$ & lots and lots & lots and lots & lots and lots \\
\hline $\begin{array}{l}\text { what type of music } \\
\text { do you enjoy most? }\end{array}$ & ii like loud rock musica & ii like loud rock musica & i like loud rock music \\
\hline $\begin{array}{l}\text { what are some of } \\
\text { your favorite games } \\
\text { to play? }\end{array}$ & i like to play cards & i like to play cards & i like to play cards \\
\hline $\begin{array}{l}\text { what has taken you } \\
\text { the longest to get } \\
\text { good or decent at? }\end{array}$ & $\begin{array}{l}\text { i worked for years to } \\
\text { perfecet my } \\
\text { photography }\end{array}$ & $\begin{array}{l}\text { i worked for years to } \\
\text { perfecet my } \\
\text { photography }\end{array}$ & $\begin{array}{l}\text { i worked for years to } \\
\text { perfect my photography }\end{array}$ \\
\hline $\begin{array}{l}\text { what food do you } \\
\text { love that a lot of } \\
\text { people might find a } \\
\text { little odd? }\end{array}$ & $\begin{array}{l}\text { very few poope can eat } \\
\text { smeked sailfish }\end{array}$ & $\begin{array}{l}\text { very few poople can eat } \\
\text { smeked sailfish }\end{array}$ & $\begin{array}{l}\text { very few people can eat } \\
\text { smoked salt fish }\end{array}$ \\
\hline $\begin{array}{l}\text { if you could start a } \\
\text { charity, what would it } \\
\text { be for? }\end{array}$ & $\begin{array}{l}\text { i woud proovide furding } \\
\text { to smal families }\end{array}$ & $\begin{array}{l}\text { i woud proovide furding } \\
\text { to small families }\end{array}$ & $\begin{array}{l}\text { i would provide funding } \\
\text { to small families }\end{array}$ \\
\hline $\begin{array}{l}\text { what advice would } \\
\text { you give to your } \\
\text { younger self? }\end{array}$ & $\begin{array}{l}\text { be patientt it wil got } \\
\text { better }\end{array}$ & $\begin{array}{l}\text { be patientt it will got } \\
\text { better }\end{array}$ & $\begin{array}{l}\text { be patient, it will get } \\
\text { better }\end{array}$ \\
\hline
\end{tabular}

\section{Supplementary Material}

Refer to Web version on PubMed Central for supplementary material.

\section{Acknowledgements}

We thank participant T5 and his caregivers for their dedicated contributions to this research, and N. Lam, E. Siauciunas, and B. Davis for administrative support. This work was supported by the Howard Hughes Medical Institute (F.R.W. and D.T.A.), Office of Research and Development, Rehabilitation R\&D Service, U.S. Department of Veterans Affairs (A2295R, N2864C); NIH: National Institute of Neurological Disorders and Stroke and BRAIN Initiative (UH2NS095548), National Institute on Deafness and Other Communication Disorders (R01DC009899, U01DC017844) (L.R.H.); NIDCD R01-DC014034, NIDCD U01-DC017844, NINDS UH2-NS095548, NINDS U01-NS098968, Larry and Pamela Garlick, Samuel and Betsy Reeves, Wu Tsai Neurosciences Institute at Stanford (J.M.H and K.V.S); Simons Foundation Collaboration on the Global Brain 543045 and Howard Hughes Medical Institute Investigator (K.V.S). The funders had no role in study design, data collection and interpretation, or the decision to submit the work for publication.

Competing Interests 
The MGH Translational Research Center has a clinical research support agreement with Neuralink, Paradromics, and Synchron, for which LRH provides consultative input. JMH is a consultant for Neuralink Corp and Proteus Biomedical, and serves on the Medical Advisory Board of Enspire DBS. KVS consults for Neuralink Corp. and CTRL-Labs Inc. (part of Facebook Reality Labs) and is on the scientific advisory boards of MIND-X Inc., Inscopix Inc., and Heal Inc. FRW, JMH, and KVS are inventors on patent application US 2021/0064135 A1 (the applicant is Stanford University), which covers the neural decoding approach taken in this work. All other authors have no competing interests.

\section{References}

1. Hochberg LR et al. Reach and grasp by people with tetraplegia using a neurally controlled robotic arm. Nature 485, 372-375 (2012). [PubMed: 22596161]

2. Collinger JL et al. High-performance neuroprosthetic control by an individual with tetraplegia. The Lancet 381, 557-564 (2013).

3. Aflalo $\mathrm{T}$ et al. Decoding motor imagery from the posterior parietal cortex of a tetraplegic human. Science 348, 906-910 (2015). [PubMed: 25999506]

4. Bouton CE et al. Restoring cortical control of functional movement in a human with quadriplegia. Nature 533, 247-250 (2016). [PubMed: 27074513]

5. Ajiboye $\mathrm{AB}$ et al. Restoration of reaching and grasping movements through brain-controlled muscle stimulation in a person with tetraplegia: a proof-of-concept demonstration. The Lancet 389, 18211830 (2017).

6. Jarosiewicz B et al. Virtual typing by people with tetraplegia using a self-calibrating intracortical brain-computer interface. Science Translational Medicine 7, 313ra179-313ra179 (2015).

7. Pandarinath $\mathrm{C}$ et al. High performance communication by people with paralysis using an intracortical brain-computer interface. eLife 6, e18554 (2017). [PubMed: 28220753]

8. Palin K, Feit AM, Kim S, Kristensson PO \& Oulasvirta A How do People Type on Mobile Devices? Observations from a Study with 37,000 Volunteers. in Proceedings of the 21st International Conference on Human-Computer Interaction with Mobile Devices and Services 1-12 (Association for Computing Machinery, 2019). doi:10.1145/3338286.3340120.

9. Yousry TA et al. Localization of the motor hand area to a knob on the precentral gyrus. A new landmark. Brain 120, 141-157 (1997). [PubMed: 9055804]

10. Willett FR et al. Hand Knob Area of Premotor Cortex Represents the Whole Body in a Compositional Way. Cell (2020) doi:10.1016/j.cell.2020.02.043.

11. Williams AH et al. Discovering Precise Temporal Patterns in Large-Scale Neural Recordings through Robust and Interpretable Time Warping. Neuron 105, 246-259.e8 (2020). [PubMed: 31786013]

12. Hinton G et al. Deep Neural Networks for Acoustic Modeling in Speech Recognition: The Shared Views of Four Research Groups. IEEE Signal Processing Magazine 29, 82-97 (2012).

13. Graves A, Mohamed A \& Hinton G Speech recognition with deep recurrent neural networks. in 2013 IEEE International Conference on Acoustics, Speech and Signal Processing 6645-6649 (2013). doi:10.1109/ICASSP.2013.6638947.

14. Xiong W et al. The Microsoft 2017 Conversational Speech Recognition System. arXiv:1708.06073 [cs] (2017).

15. He Y et al. Streaming End-to-end Speech Recognition for Mobile Devices. in ICASSP 2019 2019 IEEE International Conference on Acoustics, Speech and Signal Processing (ICASSP) 63816385 (2019). doi:10.1109/ICASSP.2019.8682336.

16. Anumanchipalli GK, Chartier J \& Chang EF Speech synthesis from neural decoding of spoken sentences. Nature 568, 493-498 (2019). [PubMed: 31019317]

17. Makin JG, Moses DA \& Chang EF Machine translation of cortical activity to text with an encoderdecoder framework. Nature Neuroscience 1-8 (2020) doi:10.1038/s41593-020-0608-8.

18. Chen $X$ et al. High-speed spelling with a noninvasive brain-computer interface. Proc Natl Acad Sci U S A 112, E6058-E6067 (2015). [PubMed: 26483479]

19. Dickey AS, Suminski A, Amit Y \& Hatsopoulos NG Single-Unit Stability Using Chronically Implanted Multielectrode Arrays. J Neurophysiol 102, 1331-1339 (2009). [PubMed: 19535480] 
20. Eleryan A et al. Tracking single units in chronic, large scale, neural recordings for brain machine interface applications. Front. Neuroeng. 7, (2014).

21. Downey JE, Schwed N, Chase SM, Schwartz AB \& Collinger JL Intracortical recording stability in human brain-computer interface users. J. Neural Eng. 15, 046016 (2018). [PubMed: 29553484]

22. Willett FR et al. Signal-independent noise in intracortical brain-computer interfaces causes movement time properties inconsistent with Fitts' law. J. Neural Eng. 14, 026010 (2017). [PubMed: 28177925]

23. Gao $\mathrm{P}$ et al. A theory of multineuronal dimensionality, dynamics and measurement. bioRxiv 214262 (2017) doi:10.1101/214262.

24. Musallam S, Corneil BD, Greger B, Scherberger H \& Andersen RA Cognitive Control Signals for Neural Prosthetics. Science 305, 258-262 (2004). [PubMed: 15247483]

25. Santhanam G, Ryu SI, Yu BM, Afshar A \& Shenoy KV A high-performance brain-computer interface. Nature 442, 195-198 (2006). [PubMed: 16838020]

26. Cunningham JP, Yu BM, Gilja V, Ryu SI \& Shenoy KV Toward optimal target placement for neural prosthetic devices. J. Neurophysiol. 100, 3445-3457 (2008). [PubMed: 18829845]

27. Pels EGM, Aarnoutse EJ, Ramsey NF \& Vansteensel MJ Estimated Prevalence of the Target Population for Brain-Computer Interface Neurotechnology in the Netherlands. Neurorehabil Neural Repair 31, 677-685 (2017). [PubMed: 28639486]

28. Vansteensel MJ et al. Fully Implanted Brain-Computer Interface in a Locked-In Patient with ALS. New England Journal of Medicine 375, 2060-2066 (2016).

29. Nijboer F et al. A P300-based brain-computer interface for people with amyotrophic lateral sclerosis. Clinical Neurophysiology 119, 1909-1916 (2008). [PubMed: 18571984]

30. Townsend $\mathrm{G}$ et al. A novel P300-based brain-computer interface stimulus presentation paradigm: Moving beyond rows and columns. Clinical Neurophysiology 121, 1109-1120 (2010). [PubMed: 20347387]

31. McCane LM et al. P300-based brain-computer interface (BCI) event-related potentials (ERPs): People with amyotrophic lateral sclerosis (ALS) vs. age-matched controls. Clinical Neurophysiology 126, 2124-2131 (2015). [PubMed: 25703940]

32. Wolpaw JR et al. Independent home use of a brain-computer interface by people with amyotrophic lateral sclerosis. Neurology 91, e258-e267 (2018). [PubMed: 29950436]

33. Bacher D et al. Neural Point-and-Click Communication by a Person With Incomplete Locked-In Syndrome. Neurorehabil Neural Repair 29, 462-471 (2015). [PubMed: 25385765]

34. Mugler EM et al. Direct classification of all American English phonemes using signals from functional speech motor cortex. J. Neural Eng. 11, 035015 (2014). [PubMed: 24836588]

35. Nurmikko A Challenges for Large-Scale Cortical Interfaces. Neuron 108, 259-269 (2020). [PubMed: 33120022]

36. Vázquez-Guardado A, Yang Y, Bandodkar AJ \& Rogers JA Recent advances in neurotechnologies with broad potential for neuroscience research. Nat Neurosci 23, 1522-1536 (2020). [PubMed: 33199897]

37. Simeral JD, Kim S-P, Black MJ, Donoghue JP \& Hochberg LR Neural control of cursor trajectory and click by a human with tetraplegia 1000 days after implant of an intracortical microelectrode array. Journal of Neural Engineering 8, 025027 (2011). [PubMed: 21436513]

38. Bullard AJ, Hutchison BC, Lee J, Chestek CA \& Patil PG Estimating Risk for Future Intracranial, Fully Implanted, Modular Neuroprosthetic Systems: A Systematic Review of Hardware Complications in Clinical Deep Brain Stimulation and Experimental Human Intracortical Arrays. Neuromodulation: Technology at the Neural Interface 23, 411-426 (2020).

39. Nuyujukian $P$ et al. Cortical control of a tablet computer by people with paralysis. PLOS ONE 13, e0204566 (2018). [PubMed: 30462658]

40. Musk E \& Neuralink. An Integrated Brain-Machine Interface Platform With Thousands of Channels. Journal of Medical Internet Research 21, e16194 (2019). [PubMed: 31642810]

41. Sahasrabuddhe K et al. The Argo: A 65,536 channel recording system for high density neural recording in vivo. bioRxiv 2020.07.17.209403 (2020) doi:10.1101/2020.07.17.209403. 
42. Sussillo D, Stavisky SD, Kao JC, Ryu SI \& Shenoy KV Making brain-machine interfaces robust to future neural variability. Nat Commun 7, (2016).

43. Dyer EL et al. A cryptography-based approach for movement decoding. Nature Biomedical Engineering 1, 967-976 (2017).

44. Degenhart AD et al. Stabilization of a brain-computer interface via the alignment of lowdimensional spaces of neural activity. Nature Biomedical Engineering 1-14 (2020) doi:10.1038/ s41551-020-0542-9. 
a
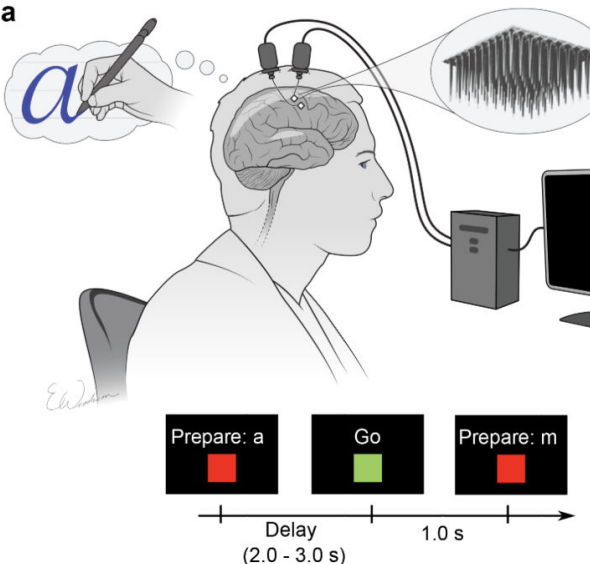

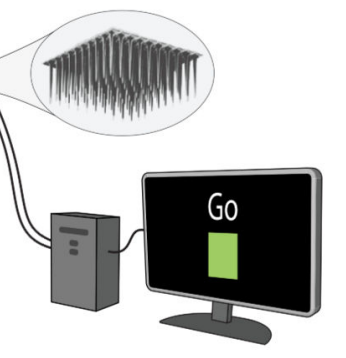

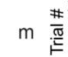

$\frac{\text { \# }}{\text { 通 }}^{27}$

b

PC

d
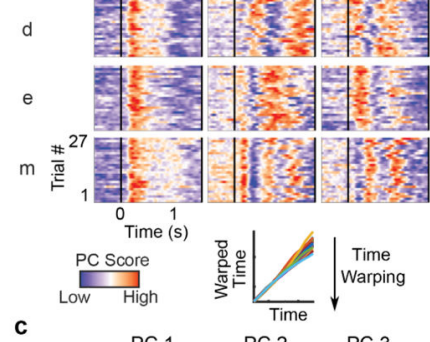

c

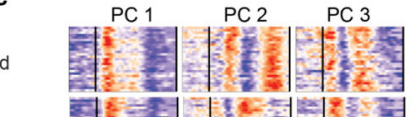

e

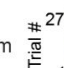

d

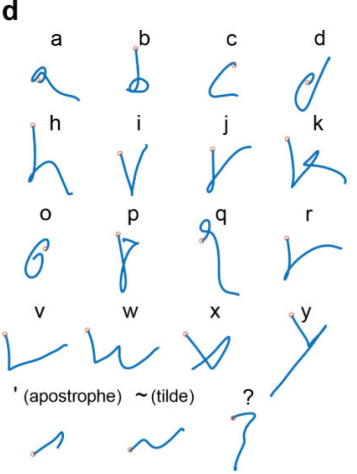

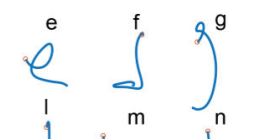

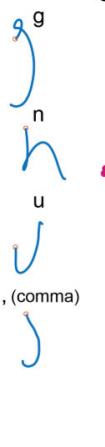

Figure 1. Neural representation of attempted handwriting.

a, To assess the neural representation of attempted handwriting, participant $\mathrm{T} 5$ attempted to handwrite each character one at a time, following the instructions given on a computer screen (lower panels depict what is shown on the screen, following the timeline). b, Neural activity in the top 3 principal components (PCs) is shown for three example letters (d, e and $\mathrm{m}$ ) and 27 repetitions of each letter ('trials'). The color scale was normalized within each panel separately for visualization. c, Time-warping the neural activity to remove trial-to-trial changes in writing speed reveals consistent patterns of activity unique to each letter. In the inset above $\mathrm{C}$, example time-warping functions are shown for the letter ' $\mathrm{m}$ ' and lie relatively close to the identity line (each trial's warping function is plotted with a differently colored line). d, Decoded pen trajectories are shown for all 31 tested characters. Intended 2D pen tip velocity was linearly decoded from the neural activity using cross-validation (each character was held out), and decoder output was denoised by averaging across trials. Orange circles denote the start of the trajectory. e, A 2-dimensional visualization of the neural activity made using t-SNE. Each circle is a single trial (27 trials for each of 31 characters). 
a

b

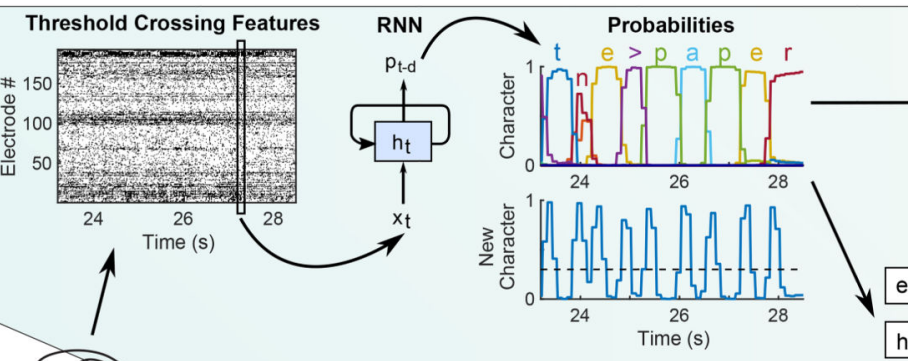
Thresholdin
(Online)

Raw Online

Output

tne paper

iterbi Search (Offline)

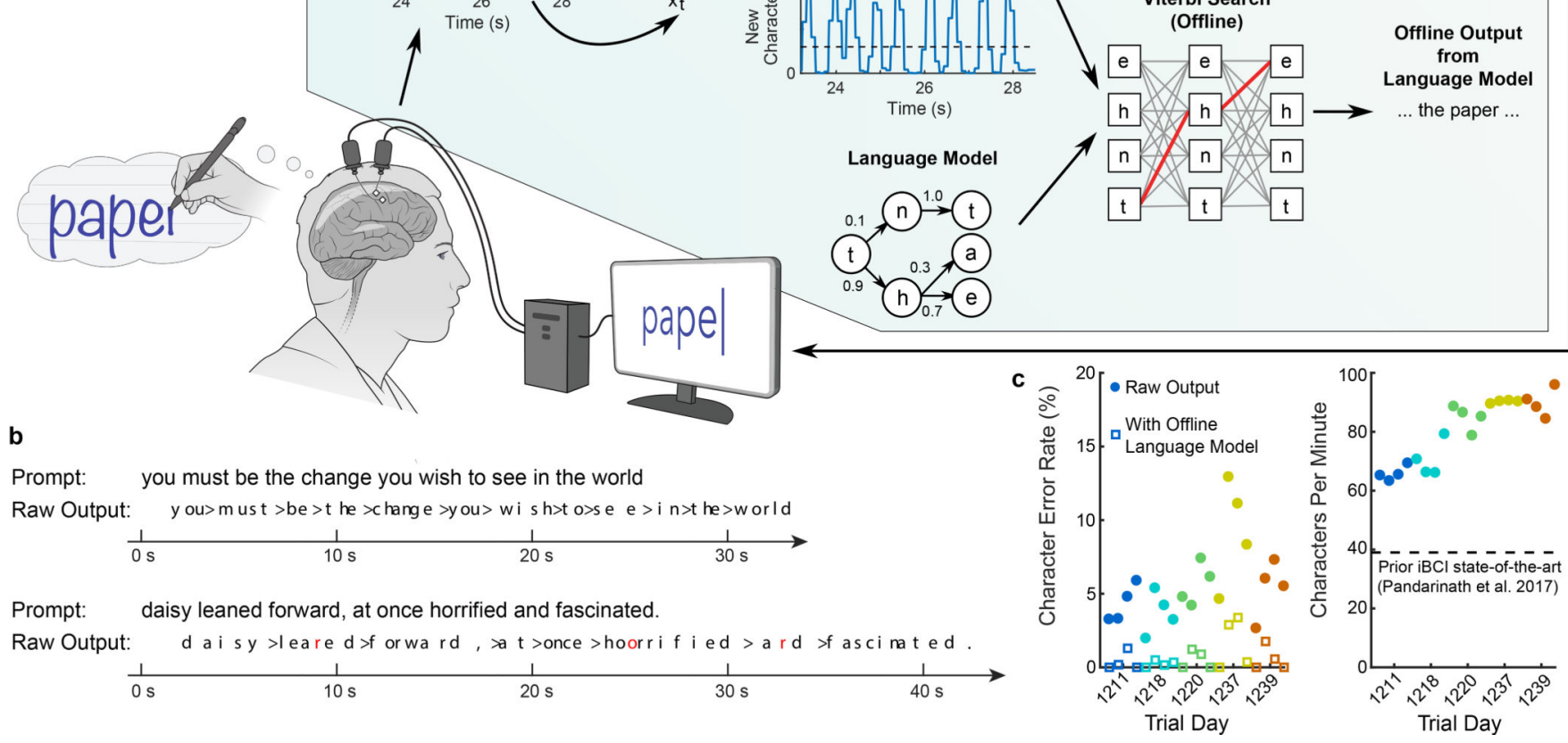

Figure 2. Neural decoding of attempted handwriting in real-time.

a, Diagram of the decoding algorithm. First, the neural activity (multiunit threshold crossings) was temporally binned and smoothed on each electrode (20 ms bins). Then, a recurrent neural network $(\mathrm{RNN})$ converted this neural population time series $\left(x_{t}\right)$ into a probability time series $\left(p_{t-d}\right)$ describing the likelihood of each character and the probability of any new character beginning. The RNN had a one second output delay (d), giving it time to observe each character fully before deciding its identity. Finally, the character probabilities were thresholded to produce "Raw Online Output" for real-time use (when the 'new character' probability crossed a threshold at time $t$, the most likely character at time $t$ $+0.3 \mathrm{~s}$ was emitted and shown on the screen). In an offline retrospective analysis, the character probabilities were combined with a large-vocabulary language model to decode the most likely text that the participant wrote (using a custom 50,000-word bigram model). b, Two real-time example trials are shown, demonstrating the RNN's ability to decode readily understandable text on sentences it was never trained on. Errors are highlighted in red and spaces are denoted with ">”. c, Error rates (edit distances) and typing speeds are shown for five days, with four blocks of 7-10 sentences each (each block is indicated with a single circle and colored according to the trial day). The speed is more than double that of the next fastest intracortical $\mathrm{BCI}^{7}$. 
a

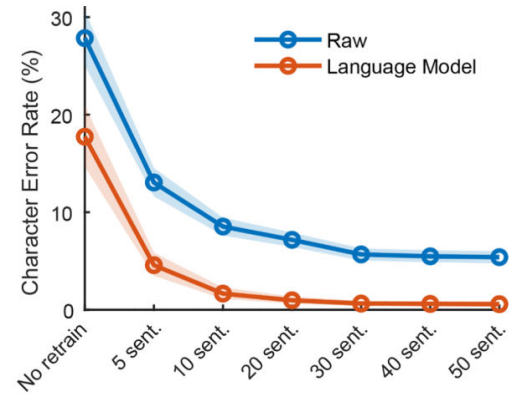

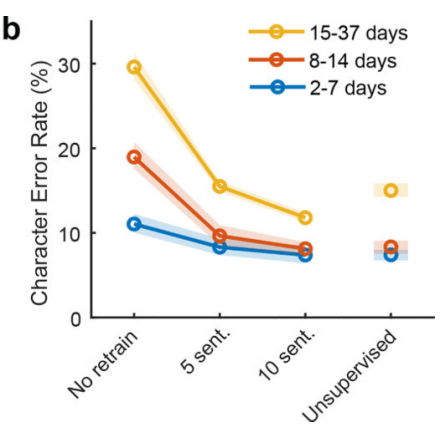

Figure 3. Performance remains high when daily decoder retraining is shortened (or unsupervised).

a, To account for neural activity changes that accrue over time, we retrained our handwriting decoder each day before evaluating it. Here, we simulated offline how decoding performance would have changed if less than the original 50 calibration sentences were used. Lines show the mean error rate across all data and shaded regions indicate $95 \%$ CIs. b, Copy typing data from eight sessions were used to assess whether less calibration data are required if sessions occur closer in time. All session pairs (X, Y) were considered. Decoders were first initialized using training data from session $\mathrm{X}$ and earlier, and then evaluated on session Y under different retraining methods (no retraining, retraining with limited calibration data, or unsupervised retraining). Lines show the average raw error rate and shaded regions indicate $95 \%$ CIs. 
a
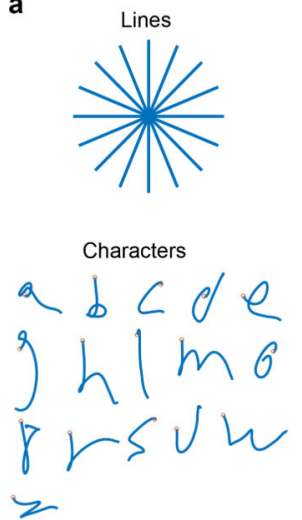

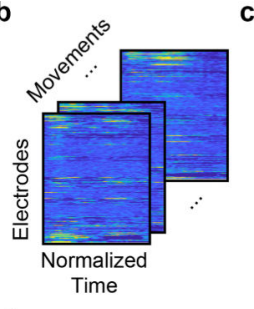

f

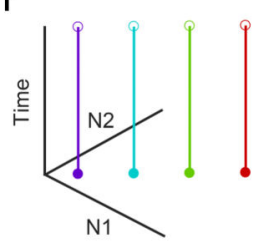

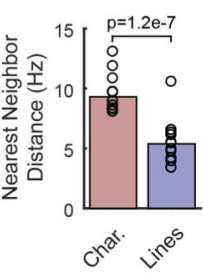
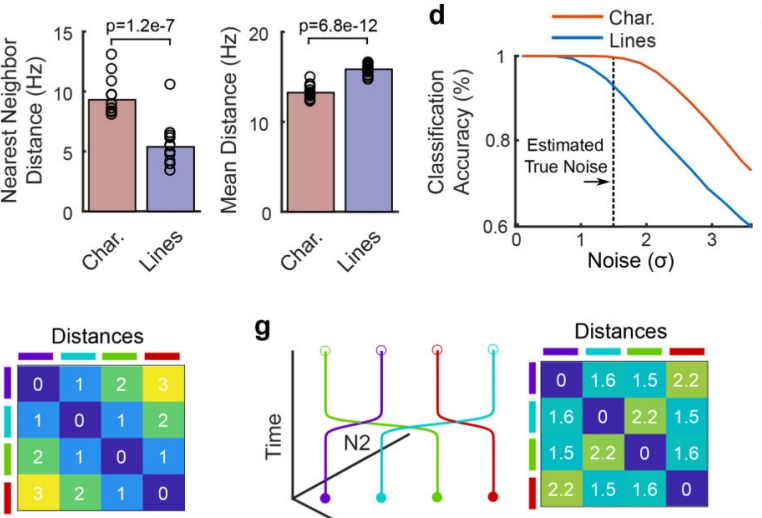

$\mathbf{g}$

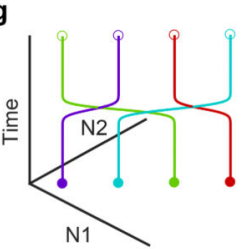

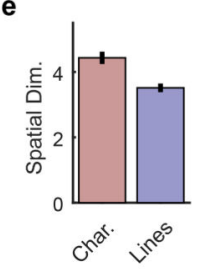

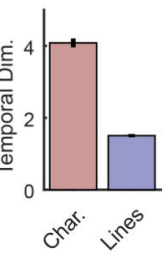

Distances

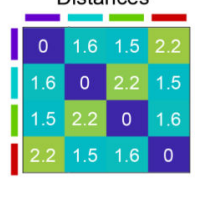

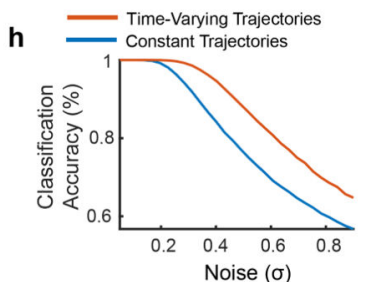

Figure 4. Increased temporal variety can make movements easier to decode.

a, We analyzed the spatiotemporal patterns of neural activity corresponding to 16 handwritten characters ( 1 second in duration) vs. 16 handwritten straight-line movements (0.6 seconds in duration). $\mathbf{b}$, Spatiotemporal neural patterns were found by averaging over all trials for a given movement (after time-warping to align the trials in time) ${ }^{11}$. Neural activity was resampled to equalize the duration of each set of movements, resulting in a 192 $\times 100$ matrix for each movement (192 electrodes and 100 time steps). c, Pairwise Euclidean distances between neural patterns were computed for each set, revealing larger nearest neighbor distances (but not mean distances) for characters. Each circle represents a single movement and bar heights show the mean. d, Larger nearest neighbor distances made the characters easier to classify than straight lines. The noise is in units of standard deviations and matches the scale of the distances in c. e, The spatial dimensionality was similar for characters and straight lines, but the temporal dimensionality was more than twice as high for characters, suggesting that more temporal variety underlies the increased nearest neighbor distances and better classification performance. Error bars show the 95\% CI. Dimensionality was quantified using the participation ratio. $\mathbf{f}-\mathbf{h}, \mathrm{A}$ toy example to give intuition for how increased temporal dimensionality can make neural trajectories more separable. Four neural trajectories are depicted (N1 and N2 are two hypothetical neurons whose activity is constrained to a single spatial dimension, the unity diagonal). Allowing the trajectories to vary in time by adding one bend (increasing the temporal dimensionality from 1 to 2 ) enables larger nearest neighbor distances (g) and better classification (h). 
Table 1.

Mean character and word error rates (with 95\% CIs) for the handwriting BCI across all 5 days.

"Raw online output" is what was decoded online (in real-time). "Online output + offline language model" was obtained by applying a language model retrospectively to what was decoded online (to simulate an autocorrect feature). "Offline bidirectional RNN + language model" was obtained by retraining a bidirectional (acausal) decoder offline using all available data, in addition to applying a language model. Word error rates can be much higher than character error rates because a word is considered incorrect if any character in that word is wrong.

\begin{tabular}{rll}
\hline & Character Error Rate [95\% CI] & Word Error Rate [95\% CI] \\
\hline Raw online output & $5.9 \%[5.3,6.5]$ & $25.1 \%[22.5,27.4]$ \\
Online output + offline language model & $0.89 \%[0.61,1.2]$ & $3.4 \%[2.5,4.4]$ \\
Offline bidirectional RNN + language model & $0.17 \%[0,0.36]$ & $1.5 \%[0,3.2]$ \\
\hline
\end{tabular}

